\title{
The Electrical Endpoint for an Electrical Fix
}

\author{
Marek Jastrzębski \\ First Department of Cardiology, Interventional Electrocardiology and Hypertension, Jagiellonian University Medical \\ College, Kraków, Poland
}

Left bundle branch block (LBBB) results in a delayed activation of the lateral wall of the left ventricle (LV). A similar LV activation pattern can be caused by right ventricular pacing or a nonspecific intraventricular conduction delay (NIVCD). These hemodynamically detrimental depolarization patterns can be fully, or partially, corrected with biventricular pacing $(\mathrm{BiV})$.

However, how do we know that the LBBB pattern was corrected with $\mathrm{BiV}$ pacing? Is the mere presence of a pacing lead in the coronary venous system enough to declare "resynchronization"? Clearly not as BiV pacing can even aggravate asynchrony. However, such an impression might be gained from the cardiac resynchronization therapy (CRT) guidelines, which do not propose any other procedural endpoint. Despite over two decades of clinical use of BiV-CRT, this method is still limited by the lack of a clear-cut endpoint or criterion that could be used during implantation and follow-up to determine if the job was done and LBBB activation pattern was fixed. Hoping for an improved prognosis when $\mathrm{BiV}$ pacing did not achieve its goal is closer to magic than medicine. We need an electrical endpoint for an electrical fix.

The QRS complex is an excellent biomarker of electrical synchrony, and the paced QRS complex during CRT

Karger@karger.com

www.karger.com/crd

(c) 2022 S. Karger AG, Basel

Karger! might indicate if resynchronization was achieved. When CRT is delivered with one of the new physiological pacing modalities, His bundle pacing (HBP-CRT) or left bundle branch area pacing (LBBAP-CRT), the situation is rather straightforward. When a complete normalization of the QRS complex is obtained and the paced QRS morphology conforms to the criteria for a normal QRS complex, then, there is no room for doubt that LBBB was corrected. For such a patient, electrical asynchrony is removed from the heart failure equation. When the paced QRS complex is not fully normalized due to the pseudodelta (HBP-CRT) or a large $\mathrm{R}$ wave in V1 (LBBAP-CRT), then, we can still base our assessment on simple QRS metrics. This is because during physiological pacing, the paced QRS complex intervals precisely correspond to the native supraventricular QRS complex intervals $[1,2]$. The time to the R-wave peak in lead V6 ( $\left.\mathrm{V}_{6} \mathrm{RWPT}\right)$, also known as the intrinsicoid deflection time, represents the time it takes for the depolarization wavefront to reach the lateral wall of the LV. This interval and its significance remain the same during HBP or LBBAP as during native supraventricular rhythm. Consequently, when the $\mathrm{V}_{6} \mathrm{RWPT}$ is over $60 \mathrm{~ms}$ (a criterion for the diagnosis of LBBB), then, we know that LBBB was not corrected. When the $\mathrm{V}_{6} \mathrm{R}$ - 
WPT is restored to normal values (i.e., $<50 \mathrm{~ms}$ ), then, we know that LBBB physiology was corrected, an elegant electrical endpoint for an electrical fix.

Alas, a similar approach for BiV-CRT is impossible as $\mathrm{BiV}$-paced QRS is often quite bizarre, broad, and far from a physiological, "healthy" QRS. Therefore, surrogate measures of restoration of physiology have to be used. The recognized alternatives are delta QRS (i.e., change in QRS duration with BiV pacing) and the disappearance of LBBB features (e.g., the presence of an $\mathrm{R}$ wave instead of the QS complex in lead V1) [3]. Delta QRS is close to be the desired, acute procedural endpoint, and it is intuitive and readily available $[4,5]$. In our own experience, we have observed that the hazard ratio for death in the longterm observation of our CRT patients shows a nearly linear relationship with the delta QRS, decreasing for QRS narrowing and increasing for QRS broadening [5]. Consequently, a lack of QRS narrowing at the end of a CRT procedure is, in our interpretation, close to a procedural failure.

However, the delta QRS has its limitations. The QRS duration measurement is not as easy as it seems as reproducibility requires experience and high precision, which is difficult to obtain without an electrophysiology system and a high-sweep speed (100-200 mm/s). Moreover, the delta QRS might not fully reflect an improvement in electrical synchrony. The often-invoked counterargument for QRS narrowing is the favorable left ventricular synchrony and hemodynamics that are observed during pure left ventricular epicardial pacing. The epicardial LV pacing, as a rule, results in QRS prolongation and a very "ugly," broad, and slurred QRS complex (and with a large QRS area). While this is most likely an exception rather than a rule, it underlines that we do not fully understand the bizarre, epicardial LV-/BiV-paced QRS complex, and we do not know how to gauge LV electrical synchrony by it.

A better alternative for the delta QRS approach might be the QRS area method, investigated by Marinko et al. [6] in this issue. Potentially, with the QRS area, more information regarding synchrony might be milked from the QRS. Although some of the limitations are similar, in both methods, the baseline RBBB morphology disturbs the relationship between the QRS duration/area reduction and outcome [5]. This is understandable; if the QRS duration or QRS area reduction is caused by the correction of a non-LV activation delay, then, it might not translate into an improved prognosis. The study by Marinko et al. [6] mainly confirms what we already know about QRS area reduction by adding credible data from

The Electrical Endpoint for an Electrical Fix yet another center. The use of digital ECGs for analysis is a major strength of this study, while the substantial dropout at the data collection stage, mainly due to the lack of post implant ECG, might have resulted in a nonrepresentative cohort. This is also suggested by the low percentage of patients with ischemic etiology of heart failure; in most CRT trials, this etiology dominates. One might also wonder about the substantial percentage of NIVCD patients. The response in NIVCD patients is generally suboptimal, and BiV pacing often prolongs the QRS complex in such patients, yet NIVCD was not a predictor of a poor outcome in this study. It would be interesting to describe the clinical response and the QRS in this sizable subgroup $(n=104)$ in greater detail and to illustrate NIVCD ECGs with a substantial QRS area reduction. Identifying NIVCD QRS patterns that are responsive to $\mathrm{BiV}$-CRT is challenging. The distinction between NIVCD and LBBB rests on the LBBB definition that is used [7]. The referenced American Heart Association (AHA) definition of LBBB [8] is burdened with imprecisions that are open to free interpretation; we do not know how these were tackled by Marinko et al. [6]. For example, the AHA definition requires "broad notched or slurred $\mathrm{R}$ waves in leads $\mathrm{I}$, aVL, V5, and V6." Literally, this means that four leads with slurred/notched QRS are necessary to diagnose LBBB. This makes the AHA definition more strict than even the Strauss LBBB definition that requires only two leads with a QRS notch/slur. However, the interpretations of the AHA definition present in the literature are varied and include the need for one notch/slur, the need for three notched/slurred leads, and a nonobligatory notch/slur. Moreover, the AHA definition does not specify if the 8 listed criteria should all be present or only some of them. It is unclear why criterion 4 forces the presence of RWPT $>60 \mathrm{~ms}$ in both V5 and V6 (the WHO definition, which is nearly identical to AHA definition, requires V5 or V6). This means that a person who has RWPT $>60 \mathrm{~ms}$ in V6 but RWPT of $55 \mathrm{~ms}$ in V5 is diagnosed with LBBB according to the WHO, but according to the AHA, this is NIVCD.

The authors concentrate their discussion on the use of this marker as a preselection tool and for the identification of potential nonresponders. I would see it more as a tool for optimal LV lead placement or optimal device programming - both aimed at maximizing the QRS area reduction. When the nonresponse is related to suboptimal LV lead position, little can be done about this after the procedure is finished.

Although BiV-CRT is probably doomed as the dominant method to deliver CRT, one can see the study by 
Marinko et al. [6] as valuable and can congratulate the authors on this important contribution that might translate into clinical practice in the near future. BiV pacing is likely to survive as the last resort option for LBBB patients in whom HBP and LBBAP fail to restore physiology. The future might be especially bright for coronary venous pacing in hybrid approaches, such as HOT-CRT and LOT-CRT $[9,10]$. The paced QRS complex assessment in these hybrid approaches might require advanced methods to determine the restoration of electrical synchrony. QRS area reduction might become one of these methods, but there a serious competitors on the horizon, like simplified body surface mapping (ECG belt) or high-fre- quency ECG $[11,12]$. Before these new methods become widely available, one would welcome both the QRS area and $\mathrm{V}_{6} \mathrm{RWPT}$ in automated ECG reports.

\section{Conflict of Interest Statement}

The author has no conflicts of interest to declare.

\section{Funding Sources}

The author has no funding sources to declare.

\section{References}

1 Jastrzebski M, Kielbasa G, Curila K, Moskal P, Bednarek A, Rajzer M, et al. Physiology-based electrocardiographic criteria for left bundle branch capture. Heart Rhythm. 2021;18:93543.

2 Jastrzebski M, Moskal P, Kukla P, Bednarek A, Kielbasa G, Rajzer M, et al. Novel approach to diagnosis of His bundle capture using individualized left ventricular lateral wall activation time as reference. J Cardiovasc Electrophysiol. 2021;32:3010-8.

3 Sweeney MO, Hellkamp AS, van Bommel RJ, Schalij MJ, Borleffs CJ, Bax JJ. QRS fusion complex analysis using wave interference to predict reverse remodeling during cardiac resynchronization therapy. Heart Rhythm. 2014;11:806-13.

4 Kronborg MB, Nielsen JC, Mortensen PT. Electrocardiographic patterns and long-term clinical outcome in cardiac resynchronization therapy. Europace. 2010;12:216-22.

5 Jastrzebski M, Baranchuk A, Fijorek K, Kisiel R, Kukla P, Sondej T, et al. Cardiac resynchronization therapy-induced acute shortening of QRS duration predicts long-term mortality only in patients with left bundle branch block. Europace. 2018;21:281-9.
6 Marinko S, Platonov P, Carlson J, Borgquist R. Baseline QRS area and reduction in QRS area are associated with lower mortality and risk of heart failure hospitalization after cardiac resynchronization therapy. Cardiology. 2022.

7 Jastrzebski M, Kukla P, Kisiel R, Fijorek K, Moskal P, Czarnecka D. Comparison of four LBBB definitions for predicting mortality in patients receiving cardiac resynchronization therapy. Ann Noninvasive Electrocardiol. 2018;23:e12563.

8 Surawicz B, Childers R, Deal BJ, Gettes LS, Bailey JJ, Gorgels A, et al. AHA/ACCF/HRS recommendations for the standardization and interpretation of the electrocardiogram: part III: intraventricular conduction disturbances: a scientific statement from the American Heart Association Electrocardiography and Arrhythmias Committee, Council on Clinical Cardiology; the American College of Cardiology Foundation; and the Heart Rhythm Society - endorsed by the International Society for computerized electrocardiology. Circulation. 2009;119:e235-40.
9 Jastrzebski M, Moskal P, Huybrechts W, Curila K, Sreekumar P, Rademakers LM, et al. Left bundle branch-optimized cardiac resynchronization therapy (LOT-CRT): results from an international LBBAP collaborative study group. Heart Rhythm. 2022;19:13-21.

10 Vijayaraman P, Herweg B, Ellenbogen KA, Gajek J. His-optimized cardiac resynchronization therapy to maximize electrical resynchronization: a Feasibility Study. Circ Arrhythm Electrophysiol. 2019;12:e006934.

11 Johnson WB, Vatterott PJ, Peterson MA, Bagwe S, Underwood RD, Bank AJ, et al. Body surface mapping using an ECG belt to characterize electrical heterogeneity for different left ventricular pacing sites during cardiac resynchronization: relationship with acute hemodynamic improvement. Heart Rhythm. 2017; 14:385-91.

12 Curila K, Prochazkova R, Jurak P, Jastrzebski M, Halamek J, Moskal P, et al. Both selective and nonselective His bundle, but not myocardial, pacing preserve ventricular electrical synchrony assessed by ultra-high-frequency ECG. Heart Rhythm. 2020;17:607-14. 